\title{
CLIPS-1D: analysis of multiple sequence alignments to deduce for residue-positions a role in catalysis, ligand-binding, or protein structure
}

\author{
Jan-Oliver Janda ${ }^{1}$, Markus Busch ${ }^{1}$, Fabian Kück ${ }^{2}$, Mikhail Porfenenko ${ }^{1}$ and Rainer Merkl ${ }^{1 *}$
}

\begin{abstract}
Background: One aim of the in silico characterization of proteins is to identify all residue-positions, which are crucial for function or structure. Several sequence-based algorithms exist, which predict functionally important sites. However, with respect to sequence information, many functionally and structurally important sites are hard to distinguish and consequently a large number of incorrectly predicted functional sites have to be expected. This is why we were interested to design a new classifier that differentiates between functionally and structurally important sites and to assess its performance on representative datasets.

Results: We have implemented CLIPS-1D, which predicts a role in catalysis, ligand-binding, or protein structure for residue-positions in a mutually exclusive manner. By analyzing a multiple sequence alignment, the algorithm scores conservation as well as abundance of residues at individual sites and their local neighborhood and categorizes by means of a multiclass support vector machine. A cross-validation confirmed that residue-positions involved in catalysis were identified with state-of-the-art quality; the mean MCC-value was 0.34 . For structurally important sites, prediction quality was considerably higher (mean $M C C=0.67$ ). For ligand-binding sites, prediction quality was lower (mean MCC $=0.12$ ), because binding sites and structurally important residue-positions share conservation and abundance values, which makes their separation difficult. We show that classification success varies for residues in a class-specific manner. This is why our algorithm computes residue-specific $p$-values, which allow for the statistical assessment of each individual prediction. CLIPS-1D is available as a Web service at http://www-bioinf. uni-regensburg.de/.

Conclusions: CLIPS-1D is a classifier, whose prediction quality has been determined separately for catalytic sites, ligand-binding sites, and structurally important sites. It generates hypotheses about residue-positions important for a set of homologous proteins and focuses on conservation and abundance signals. Thus, the algorithm can be applied in cases where function cannot be transferred from well-characterized proteins by means of sequence comparison.
\end{abstract}

\section{Background}

It is of general interest to identify important sites of a protein, for example when elucidating the reaction mechanism of an enzyme. To support this task, classifiers have been developed, which utilize different kinds of information about the protein under study. Some algorithms are based on sequences [1-11], other ones

\footnotetext{
* Correspondence: Rainer.Merkl@biologie.uni-regensburg.de 'Institute of Biophysics and Physical Biochemistry, University of Regensburg, 93040 Regensburg, Germany

Full list of author information is available at the end of the article
}

make use of 3D-data [12,13], and a third class combines both approaches [14-18].

A strong argument in favor of sequence-based methods is their broad applicability and their potential to characterize proteins with a novel fold. Additionally, some signals seem to be more pronounced in sequence- than in 3Dspace [19]. Commonly, these methods depend on a multiple sequence alignment (MSA) composed of a sufficiently large number of homologs. Based on the assumption that critical residues are not altered during evolution, the canonical feature to identify important residue-positions in an MSA is the conservation of individual columns. The

\section{Biomed Central}


degree of conservation can help to predict a role: In many cases, strictly conserved residues are essential for protein function $[7,20,21]$. In contrast, a prevalent but not exclusively found amino acid is often important for protein stability [22,23], which similarly holds for ligand-binding sites. Thus, for a precise discrimination, several properties have to be interpreted. Features that improve prediction of functionally important sites are the conservation of proximate residues $[7,24]$ and the abundance of amino acid residues observed at catalytic sites $[8,24]$. In addition, implicit features deduced from protein sequences have been utilized, like the predicted secondary structure and the predicted solvent accessible surface of residues $[5,8]$.

Most of the existing algorithms focus on the identification of sites relevant for protein function. In order to broaden the classification spectrum, we implemented the sequence-based algorithm CLIPS-1D, which predicts functionally important sites in addition to residue-positions crucial for protein structure in a mutually exclusive manner. It is based on a multiclass support vector machine, which assesses not more than seven properties deduced from residue-positions and their local neighborhood in sequence space. Our approach compares favorably with state-of-the-art classifiers and predicts catalytic residuepositions with a mean MCC-value of 0.34 . The mean MCC-value is for structurally important sites 0.67 and for ligand-binding sites it is 0.12 . Our findings show that separating ligand-binding sites and structurally important sites is difficult due to their similar properties and that classification quality depends on the residue type.

\section{Results and discussion}

Analysis of local conservation and abundance signals allows for a state-of-the-art classification

High-quality datasets consisting of catalytic sites, ligandbinding sites, and sites important for protein structure are required to train and assess support vector machines (SVMs), which predict the respective roles of residuepositions. Based on the content of EBI-databases, we prepared the redundancy-free and non-overlapping sets CAT_sites and LIG_sites, which consist of 840 catalytic sites and 4466 ligand-binding sites deduced from a set of 264 enzymes named ENZ (see Methods). Whereas the full set of functionally important sites is known for many enzymes, residues that crucially determine structure have not been identified for a representative set of proteins. Thus, to compile such sites, we had to follow an indirect approach [25] by assuming that residues in the core of proteins lacking enzymatic function are conserved due to their relevance for structure. This notion is supported by the fact that conserved hydrophobic core-residues can contribute substantially to protein stability [26]. By reannotating a comprehensive set of non-enzymes from reference [27], we culled the dataset $N O N_{-} E N Z$, which consists of 136 proteins. NON_ENZ contains 3703 buried residue-positions, which are more conserved than the mean (see Methods); we designated these sites STRUC_sites. For all proteins under study, MSAs were taken from the HSSP database [28] and filtered prior to analysis.

Next, we identified features, which allow for a state-ofthe-art classification of CAT_sites, LIG_sites, and STRUC_sites. Thus, we trained three two-class (2C-) SVMs to predict for each residue-position $k$, whether it is important for catalysis $\left(S V M_{C A T}\right)$, ligand-binding $\left(S V M_{L I G}\right)$, or protein structure $\left(S V M_{S T R U C}\right)$ and compared performance values. In the end, the features used to characterize each $k$ were in the case of $S V M_{C A T}$ a normalized Jensen-Shannon divergence cons $_{J S D}(k)$ (formula (4)) and an abundance-value abund( $k, C A T \_$sites) scoring the occurrence of residues at $C A T$ _sites according to formula (6). The proximity of $k$ was assessed by means of a weighted score cons $_{n e i b}(k)$ (formula (5)) and a novel abundance-value $a_{b u n d} d_{n e i b}\left(a a_{s}^{k}, C A T\right.$ sites $)$, deduced from conditional frequencies in the \pm 3 neighborhood [8] of CAT_sites (formula (7)). Thus, abund ${ }_{n e i b}\left(a a_{s}^{k}, C A T \_s i t e s\right)$ compares the local environment of site $k$ with the one observed for residues $a a_{s}^{k}$ at positions annotated as catalytic sites. In order to quantify the contribution of individual features to classification quality, performance was determined for SVMs exploiting either all four features or a combination of three features, respectively. Analogously, scores for LIG_sites were computed, and SVM $M_{L I G}$ was trained and assessed.

It is difficult to unambiguously determine a classifier's performance, if the numbers of positive and negative cases differ to a great extent, as is here the case. This is why we computed a battery of performance values, which are given in Additional file 1: Table S1. Their comparison confirms for our problem that the performance measures support each other, thus we focus on MCC-values [29], which are also listed in Table 1. The MCC-values for $S V M_{C A T}$ and $S V M_{L I G}$ were 0.324 and 0.213 , respectively. MCC-comparison makes clear that for CAT_sites and $L I G \_s i t e s$ all four features add to classification quality. For CAT_sites, cons $_{J S D}(k)$ and $\operatorname{abund}\left(k, C A T \_s i t e s\right)$ contributed most, for LIG_sites, the conservation score $\operatorname{cons}_{J S D}(k)$ was most relevant; compare Additional file 1: Table S1 and Additional file 1: Figure S1, which shows ROC and PROC curves.

Can $S V M_{C A T}$ and $S V M_{L I G}$ compete with state-of-the-art classifiers? For the assessment, we selected FRpred, which has outperformed other approaches and which additionally exploits the predicted secondary structure and solvent accessibility [8]. It has reached $40 \%$ precision at $20 \%$ sensitivity for the identification of catalytic 
Table 1 Classification performance of SVMs and FRpred on functionally and structurally important residuepositions

\begin{tabular}{lccc}
\hline & CAT_sites & LIG_sites & STRUC_sites \\
\hline 2C-SVM & 0.324 & 0.213 & 0.782 \\
\hline CLIPS-1D & 0.337 & 0.117 & 0.666 \\
\hline FRpred, score $\geq 8$ & 0.231 & 0.219 & $41 \%$ \\
\hline FRpred, score $=9$ & 0.250 & 0.197 & $22 \%$
\end{tabular}

The line "2C-SVM" gives MCC-values resulting from a classification of catalytic sites (CAT_sites) with $S V M_{C A T}$, of ligand-binding sites (LIG_sites) with $S V M_{L I G}$ and of structurally important sites (STRUC_sites) with SVM STRUC. The line "CLIPS$1 D^{\prime \prime}$ shows the performance of the MC-SVM. For FRpred, performance resulting from the analysis of HSSP-MSAs is given. For CAT_sites and LIG_sites, MCC-values are listed resulting from FRcons-cat or FRcons-lig scores of at least 8 or 9 , respectively. For STRUC_sites, the same percentage of false positives resulted from FRcons-cat and FRcons-lig predictions.

residues and is accessible as a Web service [8]. FRpred lists two subtypes of predictions, FRcons-cat for catalytic sites and FRcons-lig for ligand-binding sites. All results are scored with values of 0-9; the higher the score, the more probable is a functional role of the residue. A classification of CAT_sites and LIG_sites with FRpred resulted in MCC-values of 0.250 (FRcons-cat) and 0.197 (FRcons-lig), when considering predictions scored 9 as positive cases. For predictions scored at least 8 , the MCC-values were 0.231 and 0.219 , respectively. Interestingly, performance was better, when we uploaded our preprocessed HSSP-MSAs than when FRpred compiled MSAs on itself (compare Additional file 1: Table S1), which indicates the high quality of these specifically filtered MSAs. In summary, the comparison of performance values for FRpred, $S V M_{C A T}$, and $S V M_{L I G}$ confirmed that the four features selected by us account for a state-of-the-art classification.

Using corresponding features and the set STRUC_sites, we analogously trained $S V M_{S T R U C}$ for the prediction of residue-positions important for structure, which gave an MCC-value of 0.761. Classification quality was determined to the greatest extent by cons $_{J S D}(k)$. When classifying without this feature, MCC was lowered to 0.346. Utilizing the feature abund $_{\text {neib }}(k, S T R U C$ _sites $)$ deteriorated performance; a higher MCC-value (0.782) was gained by an SVM trained on the remaining three features. Even abund ( $k$, STRUC_sites) had only a marginal effect, although the respective scores differ considerably from those of abund ( $k, C A T \_$sites) and abund( $\left.k, L I G \_s i t e s\right)$; compare Table 2 and Additional file 1: Figure S2. Thus, in proteins without enzymatic function, the assessment of conservation contributed most to separate the conserved buried residues from all other ones, which constitute the negative cases. FRpred predicted with score $922 \%$ and with score $841 \%$ of the STRUC_sites as catalytic sites or ligand-binding sites; see Table 1.
Table 2 abund( $k$, CLASS)-values for amino acid residues

\begin{tabular}{|c|c|c|c|}
\hline Residue & CAT_sites & LIG_sites & STRUC_sites \\
\hline A & -2.0424 & -0.3537 & -0.1210 \\
\hline$C$ & 1.3255 & 0.7376 & 1.2398 \\
\hline D & 1.1178 & 0.0426 & -0.0498 \\
\hline$E$ & 0.6536 & -0.3856 & -0.6615 \\
\hline $\mathrm{F}$ & -0.7708 & -0.0081 & 0.5057 \\
\hline G & -0.7533 & 0.4195 & 0.7020 \\
\hline $\mathrm{H}$ & 1.8883 & 0.8279 & -0.3044 \\
\hline । & -2.8164 & -0.3026 & -0.6449 \\
\hline K & 0.6051 & -0.3615 & -1.0215 \\
\hline$L$ & -2.4503 & -0.5416 & 0.2116 \\
\hline$M$ & -1.4026 & 0.1374 & -0.4882 \\
\hline $\mathrm{N}$ & -0.1972 & 0.3566 & -0.2254 \\
\hline$P$ & -5.0000 & -0.4542 & 0.3643 \\
\hline Q & -0.7243 & -0.1841 & -0.5615 \\
\hline$R$ & 0.6834 & 0.3879 & -0.2593 \\
\hline$S$ & 0.0027 & -0.0125 & -0.7006 \\
\hline$T$ & -0.5435 & 0.2314 & -0.3363 \\
\hline V & -2.9568 & -0.4130 & -0.3294 \\
\hline W & 0.1927 & 0.5548 & 1.2811 \\
\hline$Y$ & 0.3265 & 0.4572 & 0.7058 \\
\hline
\end{tabular}

The score-values were deduced from residues belonging to the respective classes. See formula (6) for a definition of the scores.

\section{CLIPS-1D: Towards a more diversified prediction of residue function}

In order to elaborate the subtle differences distinguishing functionally and structurally important residue-positions, all combinations of the above training sets have to be exploited. This is why we prepared a multi-class support vector machine (MC-SVM) for CLIPS-1D, which was trained on the four classes CAT_sites, LIG_sites, STRUC_sites, and NOANN_sites, i.e., all residue-positions from NON_ENZ not selected as STRUC_sites. Due to the above findings on $2 \mathrm{C}-\mathrm{SVM}$, we chose the following seven features: $\operatorname{cons}_{J S D}(k), \operatorname{cons}_{n e i b}(k)$, abund $\left(k, C A T_{-}-\right.$ sites), abund(k, LIG_sites), abund(k, STRUC_sites), abund $_{\text {neib }}\left(k, C A T_{-}\right.$sites $)$, and abund $_{\text {neib }}\left(k, L I G \_s i t e s\right)$. The MC-SVM outputs a list of four class-specific probability values $p_{\text {class }}$. Based on the largest $p_{\text {class }}$-values, residuepositions were assigned one of the four classes; the resulting distributions are shown in Figure 1. 65\% of the CAT_sites and $76 \%$ of the STRUC_sites were correctly assigned. $64 \%$ of the LIG_sites and 19\% of NOANN_sites were misclassified, and each class contributed a noticeable fraction of false positives. $13 \%$ of the STRUC_sites were classified as CAT_sites and $10 \%$ as LIG_sites. Although the algorithm frequently failed to assign the correct class, separating positions with and without a crucial role was more successful: $96 \%$ of the CAT_sites, 


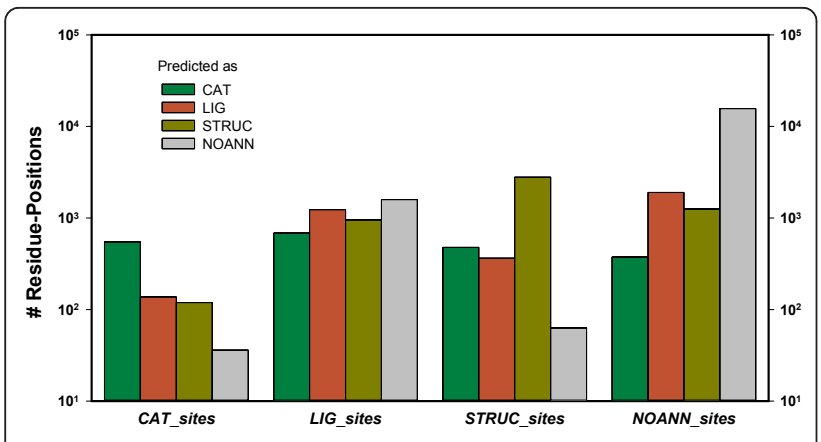

Figure 1 Classification performance of CLIPS-1D in predicting functionally and structurally important residue-positions. Based on the maximal class-probability $p_{\text {class }}$ all members of the classes CAT_sites, LIG_sites, STRUC_sites, and NOANN_sites were categorized. NOANN_sites are all residue-positions not selected as STRUC_sites in the NON_ENZ dataset, i.e. positions without assigned function. Note that the absolute numbers of residue-positions are plotted with a logarithmic scale.

65\% of the LIG_sites, and 98\% of the STRUC_sites were classified as structurally or functionally important and $81 \%$ of the NOANN_sites were classified as having no crucial function. It turned out that the respective $\mathrm{MCC}$ value was optimal, if $C A T$ _sites with $p_{C A T}(k)>0.61$ were selected as positives. In summary, the corresponding MCC-values were $0.337,0.117$, and 0.666 for CAT_sites, LIG_sites, and STRUC_sites; see Table 1. In comparison with 2C-SVMs, the performance on CAT_sites improved moderately. However, the performance on LIG_sites and STRUC_sites dropped, which indicates that the separation of LIG_sites and STRUC_sites is difficult.

The comparison of abund()-values (compare Table 2) makes clear that residues are unevenly distributed among the classes, which must influence the residue-specific classification quality. Thus, we determined class-specific MCC-values for each residue, which are listed in Table 3. As expected, performance differs drastically for individual residues and between classes. Among CAT_sites, Arg, Asp, Cys, His, Lys, and Ser were predicted with high quality. Most of the other MCC-values were near zero and no MCC-value could be computed for Pro and Val due to empty sets. The performance-values for LIG_sites were generally lower. Among STRUC_sites, the mean MCCvalue for the hydrophobic residues Ala, Ile, Leu, Met, Phe, Pro, Trp, and Val was 0.733 ; the mean of all hydrophilic ones was 0.494 . In summary, these findings proposed to determine classification quality in more detail by computing class- and residue-specific $p$-values (see Methods). Thus, the user can assess the statistical significance of each individual prediction. Table 4 lists the resulting performance for $p$-value cut-offs of $0.01,0.025$, and 0.05 . As can be seen, specificity is high in all cases; sensitivity and precision are lower and class-dependent.
Table 3 Residue-specific MCC-values

\begin{tabular}{|c|c|c|c|}
\hline Residue & CAT_sites & LIG_sites & STRUC_sites \\
\hline A & -0.002 & 0.164 & 0.774 \\
\hline$C$ & 0.404 & 0.162 & 0.676 \\
\hline D & 0.302 & 0.016 & 0.315 \\
\hline E & 0.345 & 0.052 & 0.348 \\
\hline $\mathrm{F}$ & 0.058 & 0.041 & 0.771 \\
\hline G & 0.024 & 0.262 & 0.591 \\
\hline $\mathrm{H}$ & 0.424 & -0.063 & 0.086 \\
\hline I & -0.001 & 0.135 & 0.701 \\
\hline K & 0.452 & 0.031 & 0.337 \\
\hline L & -0.001 & 0.056 & 0.815 \\
\hline M & -0.002 & 0.127 & 0.666 \\
\hline $\mathrm{N}$ & 0.071 & 0.139 & 0.561 \\
\hline$P$ & - & 0.139 & 0.683 \\
\hline Q & 0.098 & 0.111 & 0.678 \\
\hline $\mathrm{R}$ & 0.287 & 0.040 & 0.319 \\
\hline$S$ & 0.307 & 0.156 & 0.595 \\
\hline$T$ & 0.055 & 0.174 & 0.682 \\
\hline V & - & 0.119 & 0.761 \\
\hline W & -0.008 & 0.007 & 0.689 \\
\hline$Y$ & 0.097 & 0.046 & 0.741 \\
\hline
\end{tabular}

The MCC-values were determined in a class- and residue-specific manner. Due to missing cases, MCC-values could not be determined for Pro and Val residues at CAT_sites.

An alternative to CLIPS-1D is the algorithm ConSeq, which predicts functionally or structurally important residue-positions but does not distinguish catalytic and ligand-binding sites. Based on the analysis of five proteins, a success rate of 0.56 has been reported [5]. In order to estimate the performance of the latest ConSeq version [30], we have uploaded one sequence for each of the first five $E N Z$ and $N O \_E N Z$ entries (see Additional file 1: Tables S3 and S4 for PDB-IDs) and used the Web server with default parameters. As ConSeq does not differentiate between catalytic sites and ligand-binding sites, the union of CAT_sites and LIG_sites was considered as positives in this case. For the combination of these residue-positions, sensitivity was 0.41 , specificity 0.84 , and precision 0.16; for STRUC_sites the values were 0.30, 0.86 , and 0.31 , respectively. A comparison of the performance values indicates that CLIPS-1D can compete with ConSeq.

\section{Utilizing CLIPS-1D as a web service}

A version of CLIPS-1D trained on the full datasets is available as a Web service at http://www-bioinf.uniregensburg.de/. Its usage requires to upload an MSA in multiple Fasta-format; the result will be sent to the user via email. 
Table 4 Performance of CLIPS-1D for different $p$-values

\begin{tabular}{|c|c|c|c|c|c|c|c|c|c|}
\hline \multirow[t]{2}{*}{ Cut-off } & \multicolumn{3}{|c|}{ Sensitivity } & \multicolumn{3}{|c|}{ Specificity } & \multicolumn{3}{|c|}{ Precision } \\
\hline & CAT & LIG & STRUC & CAT & LIG & STRUC & CAT & LIG & STRUC \\
\hline 0.010 & 0.170 & 0.030 & 0.225 & 0.996 & 0.991 & 0.991 & 0.316 & 0.176 & 0.827 \\
\hline 0.025 & 0.276 & 0.077 & 0.445 & 0.992 & 0.977 & 0.977 & 0.270 & 0.178 & 0.789 \\
\hline 0.050 & 0.401 & 0.137 & 0.582 & 0.987 & 0.954 & 0.961 & 0.246 & 0.165 & 0.742 \\
\hline
\end{tabular}

The three performance measures were determined (see Methods) by selecting as positive cases all residue-positions with a $p$-value not greater than the given cut-off. Labels: "CAT" CAT_sites, "LIG" LIG_sites, "STRUC" STRUC_sites.

To illustrate the application of CLIPS-1D, we present an analysis of the enzyme indole-3-glycerol phosphate synthase (IGPS), which is found in many mesophilic and thermophilic species. IGPS belongs to the large and versatile family of $(\beta \alpha)_{8}$-barrel proteins, which is one of the oldest folds [31]. Additionally, folding kinetics [32] and 3D-structure of IGPS $[33,34]$ have been studied in detail.

We analyzed the HSSP-MSA related to PDB-ID 1A53, i.e. the IGPS from Sulfolobus solfataricus. Table 5 lists all CLIPS-1D predictions with a $p$-value $\leq 0.025$. According to the respective PDB-sum page [35], E51, K53, K110, E159, N180, and S211 are the catalytic residues. Besides N180, which was predicted as LIG_site, the other 5 sites were correctly identified as $C A T_{-}$sites. The sites which have contact to the ligand were classified as follows: CAT_sites E210, LIG_sites I232, STRUC_sites F112, L131, L231, NOANN_sites G212, G233, S234. Classified as LIG_sites were also K55, I179, and S181, which are all neighbors of catalytic sites. 20 residues were predicted as STRUC_sites; Figure 2 shows that all belong to the core of the protein. Their function will be discussed below.

\section{Strengths and weaknesses of CLIPS-1D}

Adding the class STRUC_sites allowed us to compare properties of functionally and structurally important residue-positions and to assess their impact on classification quality.

For CAT_sites, the abundance scores indicate a strong bias of Arg, Asp, Glu, His, and Lys towards catalytic residue-positions, which is in agreement with previous findings [24]. CAT_sites, which were classified as structurally important, were most frequently Cys and Tyr residues. Both residues are not exceedingly overrepresented at catalytic sites and abund( $k$, CAT_sites)- and abund( $k$, STRUC_sites)-values are similarly high; compare Table 2. For extracellular proteins, structurally important Cys residues are frequently involved in disulphide bonds. Thus, algorithms like DISULFIND [40] can help to clarify CLIPS-1D's Cys classification.

Least specific was the classification of LIG_sites, which also suffered the most drastic loss of performance. The MCC-value dropped from 0.21 (gained with $S V M_{L I G}$ ) to 0.12 , and most misclassifications gave STRUC_sites, which is due to the similarity of these sites with respect to the features used for classification: For both classes, $\operatorname{cons}_{I S D}(k)$ is most relevant for classification success, and among all combinations of abundance-values the pairs abund( $k$, LIG_sites) and abund( $k$, STRUC_sites) differ least; compare Table 2. The similarity of these residue-positions is further confirmed by the large number of STRUC_sites classified as functionally important by FRpred, which additionally suggests that the assessment of the predicted secondary structure and the predicted solvent accessibility contributes little to discriminate functionally and structurally important sites. It follows that LIG_sites and STRUC_sites span a fuzzy continuum, which cannot be divided by means of the considered sequence-based features. On the other hand, each MCC-value characterizes a binary classification and underestimates the performance of CLIPS-1D. For example, when assessing the performance of LIG_sites via an MCC-value, residue-positions classified as STRUC_sites were counted as false-negatives. A more detailed analysis of Figure 1 and the findings on sIGPS illustrate that LIG_sites were often classified as CAT_sites or STRUC_sites and not as sites without any function (NOANN_sites), which is a drastic difference not considered by an MCC-value.

For STRUC_sites, the MCC-value decreased from 0.78 to 0.67 for the above reasons; however, the MCC-value is still considerably high. Can one make plausible, why these buried residue-positions are preferentially occupied by a specific set of residues? At mean, hydrophobic interactions contribute $60 \%$ and hydrogen bonds $40 \%$ to protein stability; for the stability of larger proteins, hydrophobic interactions are even more important [41]. The fraction of misclassified hydrophobic STRUC_sites was low; compare MCC-values of Table 3. Thus, CLIPS-1D identifies with high reliability conserved residues of the protein's core, which are most likely important for protein stability. On the other hand, the analysis of abund(k, STRUC_sites)values (compare Table 2) shows that not all STRUC_sites are conserved hydrophobic residues: The hydrophobic residues Ala, Ile, Met, and Val are underrepresented, whereas the hydrophilic residues Cys, Gly, and Tyr are overrepresented. Additionally, the comparison of abundance scores indicates a preference of Leu, Phe, and Pro for structurally relevant sites. These preferences reflect the specific function of these residues for secondary structure 
Table 5 CLIPS-1D predictions for residue-positions in sIGPS (PDB-ID 1A53)

\begin{tabular}{|c|c|c|c|c|c|c|c|c|c|}
\hline \multirow[t]{2}{*}{ Residue } & \multirow[t]{2}{*}{ Position } & \multirow[t]{2}{*}{$p_{C A T}$} & \multirow[t]{2}{*}{$p_{\text {LIG }}$} & \multirow[t]{2}{*}{$p_{\text {StRUC }}$} & \multirow[t]{2}{*}{$p_{\text {NOANN }}$} & \multirow[t]{2}{*}{$p$-value } & \multicolumn{3}{|c|}{ Classification } \\
\hline & & & & & & & CS & LBS & STRUC \\
\hline 1 & 49 & 0.001 & 0.154 & 0.824 & 0.022 & 0.003 & & & SC \\
\hline$E$ & 51 & 0.806 & 0.075 & 0.114 & 0.005 & 0.020 & CAT & & \\
\hline K & 53 & 0.835 & 0.065 & 0.088 & 0.012 & 0.004 & CAT & & \\
\hline K & 55 & 0.051 & 0.544 & 0.197 & 0.208 & 0.011 & & SC & \\
\hline$S$ & 56 & 0.017 & 0.170 & 0.801 & 0.012 & 0.004 & & & SC \\
\hline $\mathrm{L}$ & 60 & 0.002 & 0.128 & 0.829 & 0.041 & 0.019 & & & $\mathrm{IA}$ \\
\hline A & 77 & 0.006 & 0.172 & 0.810 & 0.011 & 0.018 & & & FC \\
\hline 1 & 82 & 0.002 & 0.259 & 0.667 & 0.073 & 0.011 & & & $S R$ \\
\hline$T$ & 84 & 0.002 & 0.111 & 0.881 & 0.007 & 0.003 & & & $\mathrm{~N}$ \\
\hline $\mathrm{L}$ & 108 & 0.006 & 0.106 & 0.863 & 0.024 & 0.012 & & & $S R$ \\
\hline K & 110 & 0.866 & 0.078 & 0.046 & 0.011 & 0.002 & CAT & & \\
\hline $\mathrm{F}$ & 112 & 0.146 & 0.053 & 0.788 & 0.014 & 0.020 & & STRUC & FC \\
\hline Q & 118 & 0.007 & 0.114 & 0.872 & 0.008 & 0.002 & & & FC \\
\hline A & 122 & 0.001 & 0.066 & 0.882 & 0.051 & 0.010 & & & FC \\
\hline A & 127 & 0.024 & 0.193 & 0.776 & 0.008 & 0.022 & & & $\mathrm{~N}$ \\
\hline $\mathrm{L}$ & 131 & 0.001 & 0.071 & 0.920 & 0.008 & 0.006 & & STRUC & SR \\
\hline$L$ & 132 & 0.004 & 0.164 & 0.794 & 0.038 & 0.023 & & & $\mathrm{SR}, \mathrm{FC}$ \\
\hline 1 & 133 & 0.005 & 0.169 & 0.790 & 0.036 & 0.005 & & & FC \\
\hline L & 137 & 0.007 & 0.151 & 0.813 & 0.029 & 0.020 & & & SC,FC \\
\hline $\mathrm{L}$ & 157 & 0.001 & 0.105 & 0.886 & 0.008 & 0.010 & & & $S C, F C$ \\
\hline$E$ & 159 & 0.899 & 0.048 & 0.050 & 0.003 & 0.005 & CAT & & \\
\hline D & 165 & 0.189 & 0.071 & 0.699 & 0.040 & 0.007 & & & $\mathrm{~N}$ \\
\hline 1 & 179 & 0.001 & 0.819 & 0.068 & 0.112 & 0.021 & & SCE & \\
\hline $\mathrm{N}$ & 180 & 0.098 & 0.770 & 0.116 & 0.016 & 0.016 & LIG & & \\
\hline $\mathrm{S}$ & 181 & 0.011 & 0.774 & 0.134 & 0.081 & 0.019 & & SCE & \\
\hline$L$ & 184 & 0.009 & 0.157 & 0.818 & 0.016 & 0.020 & & & IA \\
\hline L & 197 & 0.003 & 0.130 & 0.818 & 0.049 & 0.020 & & & $\mathrm{~N}$ \\
\hline$E$ & 210 & 0.866 & 0.059 & 0.068 & 0.007 & 0.008 & & CAT & \\
\hline$S$ & 211 & 0.738 & 0.168 & 0.087 & 0.007 & 0.005 & CAT & & \\
\hline $\mathrm{L}$ & 231 & 0.003 & 0.224 & 0.762 & 0.011 & 0.025 & & STRUC & SC \\
\hline I & 232 & 0.006 & 0.835 & 0.059 & 0.099 & 0.017 & & LIG & \\
\hline
\end{tabular}

The first two columns give the residue and its position in sIGPS. The following four columns list the probabilities for the residue's membership with CAT_sites, LIG_sites, STRUC_sites, or NOANN_sites. The column labeled " $p$-value" lists the $p$-value for the class with max(p $p_{\text {CLASS) }}$. The columns "CS" and "LBS" indicate the classification of known catalytic and ligand-binding sites. The last column lists the annotation deduced for residues predicted as STRUC_sites. Meaning of labels: "CAT", "LIG", "STRUC", residues predicted as CAT sites, LIG sites, or STRUC sites, respectively. "SC" element of a stabilization center pair in sIGPS, "SCE" ditto in elGPS, "SR" stabilization residue in sIGPS; see [36]. "FC" element of the folding core; see [37]. "IA" interaction with substrate; see [38]. "N" no function assigned.

[42]. Additionally, the score-values demonstrate that CLIPS-1D does not exclusively select ILV-residues, which are considered important for protein folding [32]. STRUC_sites, misclassified as catalytic ones, were often Arg, Asp, and Glu, which shows that the abund( $k$, $C A T_{-}-$ sites)-values have a strong effect on classification. NOANN_sites predicted as CAT_sites were frequently Arg, Asp, and His; Gly, Ser, and Thr were often predicted as LIG_sites. Most likely, at least some of these residue-positions belong to binding sites on the protein-surface e.g. protein-protein interfaces. Identifying these residues is possible [43], but beyond the scope of this study.

\section{STRUC_sites are crucial elements of the sIGPS structure}

A detailed comparison of the two thermostable variants sIGPS from $S$. solfataricus [33], tIGPS from Thermotoga maritima, and the thermolabile eIGPS from Escherichia coli has made clear that these thermostable proteins have 7 strong salt bridges more than eIGPS, and that only 3 of 17 salt bridges in tIGPS and sIGPS are 


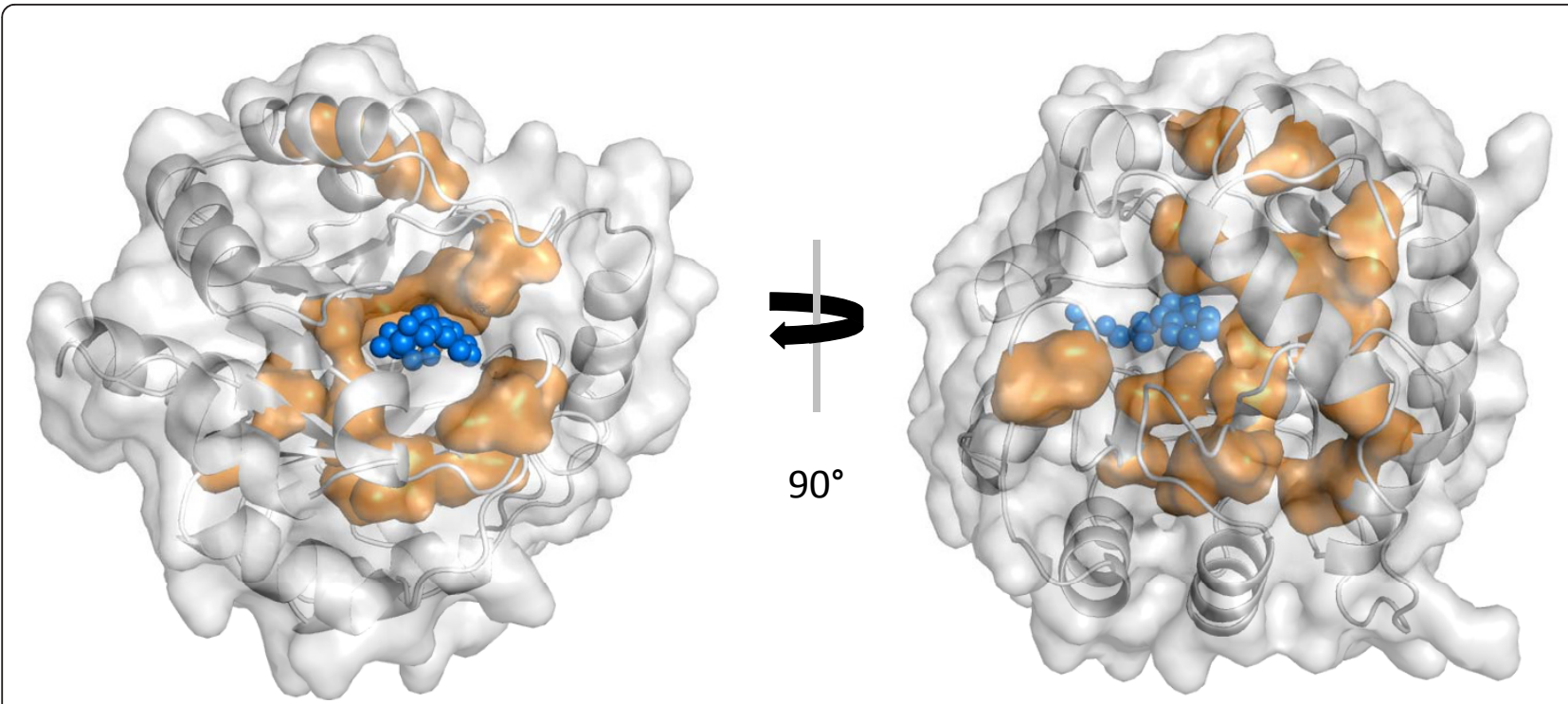

Figure 2 Localization of STRUC_sites in sIGPS. Based on PDB-ID 1A53, the surface of the whole protein (grey) and of residues predicted as STRUC_sites (orange) is shown. The substrate indole-3-glycerole phosphate is plotted in dark blue. The picture was generated by means of PyMOL [39].

topologically conserved [44]. It follows that CLIPS-1D can only identify the specific subset of structurally important residue-positions which are relevant for most of the homologous proteins constituting the MSA under study. For sIGPS, tIGPS, and eIGPS stabilization centers $(S C)$ and stabilization residues $(S R)$ have been determined [36]. Residues of $S C s$ form tight networks of cooperative interactions which are energetically stabilized; SRs are embedded into a conserved hydrophobic 3D-neigborhood. 20 residue-positions of sIGPS were classified as STRUC_sites by CLIPS-1D. 9 of these 20 residue-positions as well as the 3 false-positive LIG_sites are a $S C$ or $S R$ residue in one of the three homologous enzymes; compare Table 5. For sIGPS, the structure of folding cores, i.e. local substructures, which form early during protein folding has been determined by means of HD exchange experiments [37]. 8 of the STRUC_sites belong to fragments, which are strongest protected against deuterium exchange ( $>84 \%$, see Table 3 in reference [37]), which indicates their significant role in the partially folded protein. A molecular dynamics study [38] and a comparison of enzyme variants [34] have made clear that two more STRUC_sites belong to loops interacting with the substrate. When combining the above findings, only 4 of the 20 STRUC_sites have no accentuated function, which confirms the relevance of these sites for the enzyme's structure.

\section{Main application of CLIPS-1D: Predicting important sites of uncharacterized proteins}

For the test cases of the CASP 7 contest, the firestar [17] and the I-TASSER [45] server have reached MCC-values of 0.7 when predicting functionally important residues; the performance of other servers has been substantially lower [17]. Both servers utilize the transfer of information from evolutionary related and well-characterized proteins. If applicable, this approach allows for a superior prediction quality. However, it fails completely if the function of homologous proteins is unknown. For such cases, methods are required that identify functionally and structurally important sites by analyzing conservation signals and propensity values. In contrast to ConSeq [5] and FrPred [8], CLIPS-1D predicts a specific role in catalysis, ligand-binding, or structure for each residue-position. The only prerequisite for its application is the existence of a sufficiently large number of homologous sequences, which can easily be combined to an MSA and which should be filtered according to our experience.

The number of genes which lack annotated homologs is huge: In mid 2011, the Pfam database [46] contained nearly 4000 domains of unknown function. Additionally, a comparison of databases for protein-coding genes and their products unravels a tremendous deficit of knowledge by indicating that function is unknown for more than $40 \%$ of all protein-coding genes [47]. These genes may code for unknown folds and novel enzymatic capabilities. However, if computational biology fails to identify function, an enormous battery of experiments have to be accomplished, due to the number of distinct enzymatic activities and other protein functions observed in Nature; see e.g. [48]. Therefore, all plausible hypotheses generated by CLIPS-1D and similar methods are of value and help to reduce the number of experimental analyses. 
One might expect that exploiting the 3D-structure of a protein contributes a lot to functional assignment. This is not necessarily the case: Structure-based algorithms have failed to outperform MSA-based approaches in predicting catalytic sites and have maximally reached the same MCC-value; see [18] and references therein. However, if 3D-data and an MSA are at hand, features deduced from structure and from homologous sequences can be utilized in a concerted manner. In addition to the above features, signals caused by correlated mutations $[3,49]$ can then be utilized to further characterize catalytic sites, which are surrounded by residues spanning a network of mutual information [50]. This is why we work on exploiting a combination of these features and the near future will show, whether this approach further improves classification quality. There is an urgent need for such methods: In mid 2011, no function has been attributed to more than $4 \%$ of the protein structures deposited in the Protein Data Bank [51].

\section{Conclusions}

By analyzing an MSA by means of CLIPS-1D, residuepositions involved in catalysis can be identified with acceptable quality. In contrast, ligand-binding sites and residue-positions important for protein structure are hard to distinguish due to their similar patterns of conservation and residue propensities. Our MC-SVM can be applied to cases where the function of all homologs is unknown. The algorithm supports the user's decisions by computing a $p$-value for each prediction.

\section{Methods}

CAT_sites and LIG_sites, datasets of catalytic and ligandbinding residue-positions

To compile a test set of functionally important sites, we processed the content of the Catalytic Site Atlas (CSA) [52]. We exclusively utilized the manually curated entries of CSA and did not consider sites that have been annotated by means of PSI-BLAST alignments. In order to eliminate redundancy of proteins, we used the PISCES server [53] with a sequence-similarity cut-off of $25 \%$. For each protein, an MSA was taken from the HSSP database [28] and selected for further analyses, if it contained at least 125 sequences. The resulting dataset consists of 264 enzymes and related MSAs, which we named ENZ. These proteins contain 840 catalytic residues, which we denominated CAT_sites. For these proteins we also deduced ligand-binding sites by exploiting PDBsum pages [35]. The resulting dataset consists of 216 proteins and contains 4466 binding sites, which we named $L I G$ _sites. The datasets CAT_sites and LIG_sites do not overlap; their content is listed in Additional file 1: Tables S2 and S3.

In order to eliminate too similar and too distant sequences which might introduce a bias, the number of identical residues ident $\left(s_{i}, s_{j}\right)$ was determined for each pair of sequences $s_{i}, s_{j}$ belonging to the same MSA. Sequences were removed until the fraction of identical residues was in the range $0.25 \leq \operatorname{ident}\left(s_{i}, s_{j}\right) \leq 0.90$. Additionally, sequences deviating from the first one in length by more than $30 \%$ were deleted.

\section{STRUC_sites, a set of conserved residue-positions in proteins lacking enzymatic function}

A set of 480 non-enzyme proteins has been compiled in reference [27]. Based on PDBsum and CSA, we re-annotated all entries and prepared a redundancy-free set of MSAs as explained above. The resulting dataset NON_ENZ consists of 136 proteins and related MSAs from HSSP with at least 50 sequences. In order to exclude residues from interfaces and other binding sites, we did not consider residue-positions lying at the protein surface by eliminating all sites with a relative solvent accessible surface area of at least 5\% (see [43] and references therein). Among the remaining sites were 3703 with a conservation value cons $s_{\text {ident }}(k)>1.0$ (see formula (2)). For lack of a more biochemically motivated classification scheme, these conserved sites were regarded as important for structure. We named this set STRUC_sites, its content is listed in Additional file 1: Table S4. We designated the complement NO_ANN sites; these are the remaining 19,223 residue-positions of the NON_ENZ dataset.

\section{Conservation of an individual site}

An instructive measure to assess conservation of a single residue-position $k$ is max_frequ $(k)$, the largest amino acid frequency $f_{k}\left(a a_{i}\right)$ observed in column $k$ of an MSA:

$$
\max \_f r e q u(k)=\max _{i=1 . .20}\left(f_{k}\left(a a_{i}\right)\right)
$$

To normalize for MSA-specific variations of conservation, we computed cons ident $(k)$, which is a $\mathrm{z}$-score deduced from max_frequ $(k)$ according to

$$
\operatorname{cons}_{\text {ident }}(k)=\frac{\max _{-} f r e q u(k)-\mu_{\text {ident }}}{\sigma_{\text {ident }}}
$$

Mean $\mu_{\text {ident }}$ and standard deviation $\sigma_{\text {ident }}$ values were determined individually for each MSA under study. An alternative conservation measure is the Jensen-Shannon divergence [8] of site $k$ :

$$
J S D(k)=H\left(\frac{f_{K}^{o b s}-f^{b a c k g r}}{2}\right)-\frac{1}{2} H\left(f_{K}^{o b s}\right)-\frac{1}{2} H\left(f^{b a c k g r}\right)
$$

$f_{K}^{o b s}$ is the probability mass function for site $k$ approximated as $f_{K}^{o b s}\left(a a_{i}\right)=f_{k}\left(a a_{i}\right)$ by the amino acid frequencies observed in the respective column $k$ of the MSA; 
the mean amino acid frequencies as found in the SwissProt database [54] were taken as background frequencies $f^{b a c k g r} . H($.$) is Shannon's entropy [55]. For$ classification, we used the $\mathrm{z}$-score $\operatorname{cons}_{J S D}(k)$ :

$$
\operatorname{cons}_{J S D}(k)=\frac{J S D(k)-\mu_{J S D}}{\sigma_{J S D}}
$$

Mean $\mu_{J S D}$ and standard deviation $\sigma_{J S D}$ values were determined individually for each MSA. For the prediction of functionally important residues, $J S D(k)$ has performed better than other conservation measures [7].

\section{Conservation of a sequence neighborhood}

To characterize the conservation of a sequence neighborhood, $\operatorname{cons}_{\text {neib }}(k)$ was computed in analogy to [8]:

$$
\operatorname{cons}_{n e i b}(k)=\frac{1}{\mid \text { Neib } \mid} \sum_{l \in \text { Neib }} w_{l} \operatorname{cons}_{J S D}(k+l)
$$

Neib $=\{-3,-2,-1,+1,+2,+3\}$ determined the set of neighboring positions. The weights were: $w_{-1}=w_{+1}=3, w_{-2}=$ $w_{+2}=2, w_{-3}=w_{+3}=1$. Note that conservation of position $k$ was not considered to compute $\operatorname{cons}_{n e i b}(k)$.

\section{Propensities of catalytic sites, ligand-binding sites, and positions important for structure}

Inspired by [24], three scores abund( $k, C L A S S)$ were computed as:

$$
\operatorname{abund}(k, C L A S S)=\sum_{i=1}^{20} f_{k}\left(a a_{i}\right) \log \frac{f^{\text {CLASS }}\left(a a_{i}\right)}{f^{b a c k g r}\left(a a_{i}\right)}
$$

$f^{\text {backgr }}\left(a a_{i}\right)$ were the above background frequencies. $f^{C L A S S}\left(a a_{i}\right)$ were the frequencies of residues from one set $C L A S S \in\left\{C A T \_\right.$sites, LIG_sites, STRUC_sites $\}$.

\section{Scoring propensities of a neighborhood}

To assess the class-specific neighborhood of a site $k$, we introduced:

$$
\operatorname{abund}_{n e i b}\left(a a_{s^{\prime}}^{k} \text { CLASS }\right)=\frac{1}{|N e i b|} \sum_{l \in \text { Neib }} \sum_{i=1}^{20} f_{k+l}\left(a a_{i}\right) \log \frac{f_{k+l}^{\text {CLASS }}\left(a a_{i} \mid a a_{s}\right)}{f^{b a c k g r}\left(a a_{i}\right)}
$$

Here, $a a_{s}^{k}$ is the amino acid $a a_{s}$ occurring at site $k$ under consideration, $f_{k+l}\left(a a_{i}\right)$ is the frequency of $a a_{i}$ at position $l$ relative to $k$ and $f_{k+l}^{C L A S S}\left(a a_{i} \mid a a_{s}\right)$ is the conditional frequency of $a a_{i}$ at the same positional offset deduced from the neighborhood of all residues $a a_{s}$ of a set $C L A S S \in\left\{C A T \_\right.$sites,LIG_sites,STRUC_sites $\}$. Neib is the \pm 3 neighborhood.

\section{Evaluating classification performance}

To assess the performance of a classification, the rates TPR (Sensitivity), FPR, Specificity, and Precision

$$
T P R=\frac{T P}{T P+F N}, F P R=\frac{F P}{F P+T N}, \text { Specificity }=\frac{T N}{T N+F P}, \text { Precision }=\frac{T P}{T P+F P}
$$

as well as ROC and PROC curves were determined [56]. For a ROC curve, depending on a cut-off for one parameter (here it is $\left.p_{\text {class }}(k)\right)$, the TPR values are plotted versus the FPR values. For a PROC curve, Precision is plotted versus TPR. As a further performance measure, the Matthews correlation coefficient (MCC) has been introduced [29]:

$$
M C C=\frac{T P \cdot T N-F P \cdot F N}{\sqrt{(T P+F N)(T P+F P)(T N+F P)(T N+F N)}}
$$

MCC-values are considered a fair measure to assess performance on unbalanced sets of positives and negatives, as observed here [57]. In all formulae, TP is the number of true positives, $T N$ the number of true negatives, FP the number of false positives and $F N$ the number of false negatives. For example, when classifying catalytic sites with $S V M_{C A T}$, positives are the selected $C A T \_$sites and negatives are all other residue-positions of the considered MSAs.

\section{Classifying by means of support vector machines}

We utilized the libsvm library [58] with a Gaussian radial basis function kernel and determined during training optimal parameters $\gamma_{R B F}$ and $C$ by means of a grid search [59]. Prior to presenting features to the SVM, they were normalized according to

$$
M C C=\frac{T P \cdot T N-F P \cdot F N}{\sqrt{(T P+F N)(T P+F P)(T N+F P)(T N+F N)}}
$$

Here, $V_{e}(k)$ is for residue $k$ the value of feature $e$, and $\min \left(V_{e}\right)$ and $\max \left(V_{e}\right)$ are the smallest and the largest value determined for this feature.

Our 2C-SVMs predict for each residue-position $k$, whether it is a catalytic site $\left(S V M_{C A T}\right)$, a ligand-binding site $\left(S V M_{L I G}\right)$, or a site important for structure $\left(S V M_{S T R U C}\right)$. Taking $S V M_{C A T}$ as an example, an a posteriori probability $p_{\text {class }}(k)$, here it is $p_{C A T}(k)$, for the label " $k$ is a catalytic site" was deduced from the distance of the feature set for $k$ and the hyperplane separating catalytic and non-catalytic residue-positions [60].

We utilized $p_{\text {class }}(k)$ to assess performance and to assign classes. Training and assessment was organized as an 8-fold cross validation. For each training step, the number of positive and negative cases was balanced, i.e. for $S V M_{C A T}$, residue-positions from CAT_sites and the same number of non-catalytic sites was selected. In order to eliminate sampling bias during the grid search, each parameter was deduced as means from training trials with the same positives and 50 different, randomly selected sets of negative cases. To compute the 
performance measures (e.g. MCC-values), all positive and all negative cases belonging to the selected subset of MSAs were classified.

Analogously, an MC-SVM was applied to the four classes CAT_sites, LIG_sites, STRUC_sites, and NOANN_sites. The output of the MC-SVM consists of four class-probabilities $p_{\text {class }}$ (see [60]) for each residueposition. These were deduced from the a posteriori probabilities of the six 2C-SVMs, which were trained on one specific combination of two classes, each. Each residue-positions $k$ was assigned to the class, whose $p_{\text {class }}{ }^{-}$ value was largest. $p$-values were determined as follows: For each class and each residue, the respective cumulative distribution was deduced from the $p_{\text {class }}$-values of all residue-positions $k$ not belonging to the considered class. I. e., the $p$-value for a Glu-residue with $p_{\text {STRUC }}$ value $s(k)$ is the fraction of all Glu-residues from NOANN_sites reaching or surpassing $s(k)$.

\section{Additional material}

Additional file 1: A plot comparing abund( $k, C L A S S)$-values, Figures and Tables giving performance-values of $2 \mathrm{C}$-SVMs, and Tables listing the composition of datasets. (PDF $327 \mathrm{~kb}$ ).

\section{Acknowledgements}

The work was supported by DFG grant ME-2259/1-1.

\section{Author details}

${ }^{1}$ Institute of Biophysics and Physical Biochemistry, University of Regensburg, 93040 Regensburg, Germany. ${ }^{2}$ Faculty of Mathematics and Computer Science, University of Hagen, 58084 Hagen, Germany.

\section{Authors' contributions}

JOJ designed and implemented algorithms, and trained and assessed the SVMs. MB, FK, and MP prepared datasets and were involved in programming and assessment. RM conceived of and coordinated the study, and wrote the manuscript. All authors read and approved the manuscript.

\section{Competing interests}

The authors declare that they have no competing interests.

Received: 22 December 2011 Accepted: 5 April 2012

Published: 5 April 2012

\section{References}

1. Overington J, Johnson MS, Sali A, Blundell TL: Tertiary structural constraints on protein evolutionary diversity: templates, key residues and structure prediction. Proc Biol Sci 1990, 241(1301):132-145.

2. Casari $G$, Sander $C$, Valencia $A$ : A method to predict functional residues in proteins. Nat Struct Biol 1995, 2(2):171-178.

3. Lichtarge O, Bourne HR, Cohen FE: An evolutionary trace method defines binding surfaces common to protein families. J Mol Biol 1996 257(2):342-358.

4. Huang JY, Brutlag DL: The EMOTIF database. Nucleic Acids Res 2001, 29(1):202-204

5. Berezin C, Glaser F, Rosenberg J, Paz I, Pupko T, Fariselli P, Casadio R, BenTal N: ConSeq: the identification of functionally and structurally important residues in protein sequences. Bioinformatics 2004, 20(8):1322-1324
6. Gutman R, Berezin C, Wollman R, Rosenberg Y, Ben-Tal N: QuasiMotiFinder: protein annotation by searching for evolutionarily conserved motif-like patterns. Nucleic Acids Res 2005, 33:W255-261, Web Server issue.

7. Capra JA, Singh M: Predicting functionally important residues from sequence conservation. Bioinformatics 2007, 23(15):1875-1882.

8. Fischer JD, Mayer CE, Söding J: Prediction of protein functional residues from sequence by probability density estimation. Bioinformatics 2008, 24(5):613-620.

9. Sankararaman S, Kolaczkowski B, Sjölander K: INTREPID: a web server for prediction of functionally important residues by evolutionary analysis. Nucleic Acids Res 2009, 37:W390-395, Web Server issue.

10. Tang K, Pugalenthi G, Suganthan PN, Lanczycki CJ, Chakrabarti S: Prediction of functionally important sites from protein sequences using sparse kernel least squares classifiers. Biochem Biophys Res Commun 2009, 384(2):155-159.

11. Erdin S, Ward RM, Venner E, Lichtarge O: Evolutionary trace annotation of protein function in the structural proteome. J Mol Biol 2010, 396(5):1451-1473.

12. Petrey $D$, Fischer $M$, Honig B: Structural relationships among proteins with different global topologies and their implications for function annotation strategies. Proc Natl Acad Sci USA 2009, 106(41):17377-17382.

13. Mitternacht $S$, Berezovsky IN: A geometry-based generic predictor for catalytic and allosteric sites. Protein Eng 2011, 24(4):405-409.

14. Panchenko AR, Kondrashov F, Bryant S: Prediction of functional sites by analysis of sequence and structure conservation. Prot Sci 2004, 13(4):884-892.

15. Laskowski RA, Watson JD, Thornton JM: ProFunc: a server for predicting protein function from 3D structure. Nucleic Acids Res 2005, 33:W89-93, Web Server issue.

16. Kalinina OV, Gelfand MS, Russell RB: Combining specificity determining and conserved residues improves functional site prediction. $B M C$ Bioinformatics 2009, 10:174

17. Lopez G, Maietta P, Rodriguez JM, Valencia A, Tress ML: Firestar-advances in the prediction of functionally important residues. Nucleic Acids Res 2011, , 39 Web Server: W235-241.

18. Yahalom R, Reshef D, Wiener A, Frankel S, Kalisman N, Lerner B, Keasar C: Structure-based identification of catalytic residues. Proteins 2011, 79(6):1952-1963.

19. Dou Y, Geng X, Gao H, Yang J, Zheng X, Wang J: Sequence conservation in the prediction of catalytic sites. Prot J 2011, 30(4):229-239.

20. Pei J, Grishin NV: AL2CO: calculation of positional conservation in a protein sequence alignment. Bioinformatics 2001, 17(8):700-712.

21. Wang K, Samudrala R: Incorporating background frequency improves entropy-based residue conservation measures. BMC Bioinformatics 2006, 7:385

22. Lehmann M, Loch C, Middendorf A, Studer D, Lassen SF, Pasamontes L, van Loon AP, Wyss M: The consensus concept for thermostability engineering of proteins: further proof of concept. Protein Eng 2002, 15(5):403-411.

23. Amin N, Liu AD, Ramer S, Aehle W, Meijer D, Metin M, Wong S, Gualfetti $P$, Schellenberger $V$ : Construction of stabilized proteins by combinatorial consensus mutagenesis. Protein Eng Des Sel 2004, 17(11):787-793.

24. Bartlett GJ, Porter CT, Borkakoti N, Thornton JM: Analysis of catalytic residues in enzyme active sites. J Mol Biol 2002, 324(1):105-121.

25. Ptitsyn $\mathrm{OB}$, Ting $\mathrm{KL}$ : Non-functional conserved residues in globins and their possible role as a folding nucleus. J Mol Biol 1999, 291(3):671-682.

26. Schueler-Furman O, Baker D: Conserved residue clustering and protein structure prediction. Proteins 2003, 52(2):225-235.

27. Davidson NJ, Wang X: Non-alignment features based enzyme/nonenzyme classification using an ensemble method. Proc Int Conf Mach Learn Appl 2010, 546-551.

28. Sander C, Schneider R: Database of homology-derived protein structures and the structural meaning of sequence alignment. Proteins 1991, 9(1):56-68.

29. Matthews BW: Comparison of the predicted and observed secondary structure of T4 phage lysozyme. Biochim Biophys Acta 1975, 405(2):442-451.

30. Ashkenazy H, Erez E, Martz E, Pupko T, Ben-Tal N: ConSurf 2010: calculating evolutionary conservation in sequence and structure of proteins and nucleic acids. Nucleic Acids Res 2010, , 38 Web Server: W529-533. 
31. Caetano-Anollés G, Kim HS, Mittenthal JE: The origin of modern metabolic networks inferred from phylogenomic analysis of protein architecture. Proc Natl Acad Sci USA 2007, 104(22):9358-9363.

32. Gu Z, Rao MK, Forsyth WR, Finke JM, Matthews CR: Structural analysis of kinetic folding intermediates for a TIM barrel protein, indole-3-glycerol phosphate synthase, by hydrogen exchange mass spectrometry and Gō model simulation. J Mol Biol 2007, 374(2):528-546.

33. Hennig M, Darimont B, Sterner R, Kirschner K, Jansonius JN: $2.0 \AA$ structure of indole-3-glycerol phosphate synthase from the hyperthermophile Sulfolobus solfataricus: possible determinants of protein stability. Structure 1995, 3(12):1295-1306

34. Schneider B, Knöchel T, Darimont B, Hennig M, Dietrich S, Babinger K, Kirschner K, Sterner R: Role of the N-terminal extension of the $(\beta a)_{8}$-barrel enzyme indole-3-glycerol phosphate synthase for its fold, stability, and catalytic activity. Biochemistry 2005, 44(50):16405-16412.

35. Laskowski RA, Chistyakov W, Thornton JM: PDBsum more: new summaries and analyses of the known 3D structures of proteins and nucleic acids. Nucleic Acids Res 2005, 33 Database: D266-268.

36. Bagautdinov B, Yutani K: Structure of indole-3-glycerol phosphate synthase from Thermus thermophilus HB8: implications for thermal stability. Acta Crystallogr D: Biol Crystallogr 2011, 67(Pt 12):1054-1064.

37. Gu Z, Zitzewitz JA, Matthews CR: Mapping the structure of folding cores in TIM barrel proteins by hydrogen exchange mass spectrometry: the roles of motif and sequence for the indole-3-glycerol phosphate synthase from Sulfolobus solfataricus. J Mol Biol 2007, 368(2):582-594

38. Mazumder-Shivakumar D, Bruice TC: Molecular dynamics studies of ground state and intermediate of the hyperthermophilic indole-3glycerol phosphate synthase. Proc Natl Acad Sci USA 2004, 101(40):14379-14384

39. Schrödinger: PyMOL. Schrödinger Inc.

40. Ceroni A, Passerini A, Vullo A, Frasconi P: DISULFIND: a disulfide bonding state and cysteine connectivity prediction server. Nucleic Acids Res 2006, , 34 Web Server: W177-181.

41. Pace CN, Fu H, Fryar KL, Landua J, Trevino SR, Shirley BA, Hendricks MM, limura S, Gajiwala K, Scholtz JM, et al: Contribution of hydrophobic interactions to protein stability. J Mol Biol 2011, 408(3):514-528.

42. Chou PY, Fasman GD: Empirical predictions of protein conformation. Annu Rev Biochem 1978, 47:251-276.

43. Zellner H, Staudigel M, Trenner T, Bittkowski M, Wolowski V, Icking C, Merkl R: Prescont: Predicting protein-protein interfaces utilizing four residue properties. Proteins 2012, 80(1):154-168.

44. Knöchel T, Pappenberger A, Jansonius JN, Kirschner K: The crystal structure of indoleglycerol-phosphate synthase from Thermotoga maritima. Kinetic stabilization by salt bridges. J Biol Chem 2002, 277(10):8626-8634.

45. Zhang Y: I-TASSER server for protein 3D structure prediction. BMC Bioinformatics 2008, 9:40.

46. Finn RD, Mistry J, Schuster-Böckler B, Griffiths-Jones S, Hollich V, Lassmann T, Moxon S, Marshall M, Khanna A, Durbin R, et al: Pfam: clans, web tools and services. Nucleic Acids Res 2006, 34:D247-D251, Database issue.

47. Friedberg I, Jambon M, Godzik A: New avenues in protein function prediction. Prot Sci 2006, 15(6):1527-1529.

48. Gerlt JA, Allen KN, Almo SC, Armstrong RN, Babbitt PC, Cronan JE, Dunaway-Mariano D, Imker HJ, Jacobson MP, Minor W, et al: The enzyme function initiative. Biochemistry 2011, 50(46):9950-9962.

49. Merkl R, Zwick M: H2r: Identification of evolutionary important residues by means of an entropy based analysis of multiple sequence alignments. BMC Bioinformatics 2007, 9:151.

50. Marino Buslje C, Teppa E, Di Domenico T, Delfino JM, Nielsen M: Networks of high mutual information define the structural proximity of catalytic sites: implications for catalytic residue identification. PLoS Comp Biol 2010, 6(11):e1000978.

51. Berman HM, Westbrook J, Feng Z, Gilliland G, Bhat TN, Weissig H, Shindyalov IN, Bourne PE: The Protein Data Bank. Nucleic Acids Res 2000, 28(1):235-242.

52. Porter CT, Bartlett GJ, Thornton JM: The Catalytic Site Atlas: a resource of catalytic sites and residues identified in enzymes using structural data. Nucleic Acids Res 2004, , 32 Database: D129-133.

53. Wang G, Dunbrack RL Jr: PISCES: recent improvements to a PDB sequence culling server. Nucleic Acids Res 2005, , 33 Web Server: W94-98.
54. Bairoch A, Apweiler R: The SWISS-PROT protein sequence database and its supplement TrEMBL in 2000. Nucleic Acids Res 2000, 28(1):45-48.

55. Shannon C: A mathematical theory of communication. Bell Sys Tech J 1948, 27:379-423.

56. Davis J, Goadrich M: The relationship between precision-recall and ROC curves. ICML NewYork: Pittsburgh; 2006, 233-240.

57. Ezkurdia I, Bartoli L, Fariselli P, Casadio R, Valencia A, Tress ML: Progress and challenges in predicting protein-protein interaction sites. Brief Bioinform 2009, 10(3):233-246.

58. Chang CC, Lin CJ: LIBSVM: a library for support vector machines. ACM Trans Int Sys Tech 2011, 2(27):1-27.

59. Schölkopf B, Smola AJ: Learning with kernels London: The MIT Press; 2002.

60. Wu TF, Lin CJ, Weng RC: Probability estimates for multi-class classification by pairwise coupling. J Mach Learn Res 2004, 5:975-1005.

\section{doi:10.1186/1471-2105-13-55}

Cite this article as: Janda et al:: CLIPS-1D: analysis of multiple sequence alignments to deduce for residue-positions a role in catalysis, ligandbinding, or protein structure. BMC Bioinformatics 2012 13:55.

\section{Submit your next manuscript to BioMed Central and take full advantage of:}

- Convenient online submission

- Thorough peer review

- No space constraints or color figure charges

- Immediate publication on acceptance

- Inclusion in PubMed, CAS, Scopus and Google Scholar

- Research which is freely available for redistribution

Submit your manuscript at www.biomedcentral.com/submit
C Biomed Central 